\title{
The Dirac Equation in Exterior Form
}

\author{
I. M. Benn ${ }^{1}$ and R. W. Tucker ${ }^{2}$ \\ 1 Department of Natural Philosophy, University of Glasgow, United Kingdom \\ 2 Department of Physics, University of Lancaster, United Kingdom
}

\begin{abstract}
Using the correspondence between the Clifford and exterior algebras we write the Dirac equation in terms of differential forms. The covariances of the theory are then examined. We show in detail the correspondence with usual matrix methods.
\end{abstract}

\section{Introduction}

Kähler [1] has used a correspondence between Clifford and exterior algebras associated with space-time to describe particles with half integer spin by means of sections of the exterior bundle over space time (inhomogeneous differential forms). In Minkowski space Kähler's equation decouples into four minimal left ideals of the Clifford algebra, and is equivalent to four identical Dirac equations [2,3]. Thus the Kähler equation is not the Dirac equation. Moreover, in an arbitrary space-time the Kähler equation does not split into minimal left ideals. These features of the Kähler equation are not inevitable consequences of using differential forms for the description of half-integer spin, indeed we here show how the Kähler equation can be modified so that, even in a curved space-time, it describes fields lying in one minimal left ideal. In this way we are lead to the Dirac equation.

We shall make contact with the usual matrix formulation of the Dirac equation by making clear how, and in what sense, the Clifford algebra is a matrix algebra. Since we regard the Clifford algebra as being embedded in the Kähler-Atiyah algebra, a basis for this matrix algebra will consist of differential forms. This differentiable basis is the main feature inherited from exploitation of the KählerAtiyah algebra. It will be shown how covariant derivatives of this basis can provide the usual "spin connection" terms in the curved space Dirac equation.

Having written the Dirac equation in terms of inhomogeneous differential forms it is natural to question the compatability of the "spinorial" nature of the equation with the "tensorial" nature of the forms. We shall argue that there are two a priori distinct covariances; a trivial GL $(4, R)$ frame covariance, and a GL $(4, R)$ 
covariance under inner automorphisms of the Clifford algebra (corresponding to change of representation of $\gamma$-matrices). However, they may be combined to reproduce the usual spinorial covariance of the matrix components of the equation.

Since the cotangent space is a real vector space we are led naturally to consider the real Clifford algebra. The doubling of degrees of freedom necessary for the inclusion of charged fields does not necessitate complexifying the Clifford algebra. We show how "charged" spinors may be described by non-minimal ideals, with the U(1) transformation generated by Clifford multiplication.

Unless otherwise stated we shall use the conventions and notation of reference [3]. For simplicity of notation we shall denote the Kähler-Atiyah algebra of the cotangent space of the space-time manifold by $\mathscr{A}$. For discussion of algebraic properties $\mathscr{A}$ is regarded as (and sometimes called) a Clifford algebra. When discussing field equations we shall regard elements of $\mathscr{A}$ as sections of the KählerAtiyah bundle over space time. Throughout we shall juxtapose symbols to denote Clifford multiplication.

\section{The Wedderburn Decomposition of $\mathscr{A}$}

Since $\mathscr{A}$ is simple, Wedderburn's principle structure theorem ensures that

$$
\mathscr{A}=\mathscr{D} \times \mathscr{M}_{m},
$$

where $\mathscr{D}$ is a division algebra and $\mathscr{M}_{m}$ is a total matrix algebra [4]. The degree of the matrix algebra, $m$, is given by the number of pairwise orthogonal primitive idempotents of $\mathscr{A}$. For our algebra $m=4$ (our space time metric has a signature with three plus and one minus sign) and

$$
\mathscr{A}=\mathbb{R} \times \mathscr{M}_{4},
$$

i.e. the algebra of real $4 \times 4$ matrices. Since we are interested in differentiating these matrices we show how the matrix and division algebras may be constructed; this essentially entails following the proof of Wedderburn's theorem.

If $P$ is a primitive idempotent of $\mathscr{A}$ then $P \mathscr{A} P=\mathscr{D}$, where $\mathscr{D}$ is the division algebra appearing in the Wedderburn decomposition (I.1). (Note that $P$ is the identity of $\mathscr{D}$.) We here have [5]

$$
P \mathscr{A} P=4 S_{0}(\mathscr{A} P) P,
$$

where $S_{p}$ projects the $p$-form component of $\mathscr{A}$.

Let $\left\{P_{i}\right\}, i=1, \ldots, 4$, be a complete set of pairwise orthogonal primitive idempotents of $\mathscr{A}$. Then the simplicity of $\mathscr{A}$ ensures $\exists c_{12}, c_{23}, c_{34} \in \mathscr{A}$ such that

$$
P_{1}=c_{12} P_{2} C_{12}^{-1}, \quad P_{2}=c_{23} P_{3} c_{23}{ }^{-1}, \quad P_{3}=c_{34} P_{4} c_{34}{ }^{-1} \text {. }
$$

Let $c_{13}=c_{12} c_{23}$ etc., giving

$$
P_{i}=c_{i j} P_{j} c_{i j}^{-1}, \quad \forall i, j=1, \ldots, 4,
$$

with $c_{i j} c_{j k}=c_{i k}$ (no sum). Let

$$
\mathbf{e}_{i j}=P_{i} c_{i j} P_{j}=P_{i} c_{i j}=c_{i j} P_{j}
$$


Then

$$
\begin{aligned}
\mathbf{e}_{i j} \mathbf{e}_{p k} & =\mathbf{e}_{i j} P_{j} P_{p} \mathbf{e}_{p k}=0 \text { for } j \neq p, \\
\mathbf{e}_{i j} \mathbf{e}_{j k} & =P_{i} c_{i j} c_{j k} P_{k}=P_{i} c_{i k} P_{k}=\mathbf{e}_{i k} .
\end{aligned}
$$

That is the $\left\{\mathbf{e}_{i j}\right\}$ have the algebra of an "ordinary" matrix basis. Completeness of the $\left\{P_{i}\right\}$ gives

$$
\begin{aligned}
\mathscr{A} & =\sum_{i j} P_{i} \mathscr{A} P_{j} \\
& =\sum_{i j} P_{i} \mathscr{A} c_{j i} P_{i} c_{j i}{ }^{-1} \\
& =\sum_{i j} 4 S_{0}\left(\mathscr{A} c_{j i} P_{i}\right) P_{i} c_{j i}{ }^{-1} \\
& =\sum_{i j} 4 S_{0}\left(\mathscr{A} c_{j i} P_{i}\right) P_{i} c_{i j}, \\
\mathscr{A} & =\sum_{i j} 4 S_{0}\left(\mathscr{A} \mathbf{e}_{j i}\right) \mathbf{e}_{i j} .
\end{aligned}
$$

If we denote the matrix components of $\mathscr{A}$ by $A_{i j}$ then

i.e. $\operatorname{Tr} \mathscr{A}=4 S_{0}(\mathscr{A})$.

$$
\begin{aligned}
\mathscr{A} & =\sum_{i j} A_{i j} \mathbf{e}_{i j}, \\
S_{0}(\mathscr{A}) & =\sum_{i j} A_{i j} S_{0}\left(\mathbf{e}_{i j}\right)=\sum_{i j} A_{i j} S_{0}\left(P_{i} c_{i j} P_{j}\right) \\
& =\sum_{i} A_{i i} S_{0}\left(P_{i}\right)=\frac{1}{4} \sum_{i} A_{i i},
\end{aligned}
$$

Transposition (denoted ${ }^{T}$ ) is an anti-involution such that

$$
\mathbf{e}_{i j}{ }^{T}=\mathbf{e}_{j i} .
$$

In this way the definition of transposition depends on a choice of matrix basis. We can relate transposition to the main anti-involution, $\xi$, by

for some $C$.

$$
\mathscr{A}^{T}=C^{-1} \xi \mathscr{A} C
$$

The main anti-involution is defined by

$$
\xi X=X \text { if } X \text { is a covector }
$$

and satisfies

$$
\xi S_{p}(\mathscr{A})=(-1)^{[p / 2]} S_{p}(\mathscr{A}),
$$

where $[p / 2]$ denotes the integer part of $p / 2$. On general grounds we must have $\xi C= \pm C$; in fact, for this algebra we have

$$
\xi C=-C \text {. }
$$

We now give an explicit example of the above.

Let

$$
\begin{aligned}
& P_{1}=\frac{1}{4}\left(1+e^{02}\right)\left(1+e^{1}\right), \\
& P_{2}=\frac{1}{4}\left(1-e^{02}\right)\left(1+e^{1}\right), \\
& P_{3}=\frac{1}{4}\left(1+e^{02}\right)\left(1-e^{1}\right), \\
& P_{4}=\frac{1}{4}\left(1-e^{02}\right)\left(1-e^{1}\right) .
\end{aligned}
$$


The $\left\{e^{a}\right\}$ is an orthonormal coframe with $\left(e^{0}\right)^{2}=-1,\left(e^{i}\right)^{2}=1, i=1,2,3$, and $e^{02} \equiv e^{0} e^{2}$ etc. Choosing $c_{12}=e^{03}, c_{34}=e^{03}, c_{13}=e^{3}$ we construct the following:

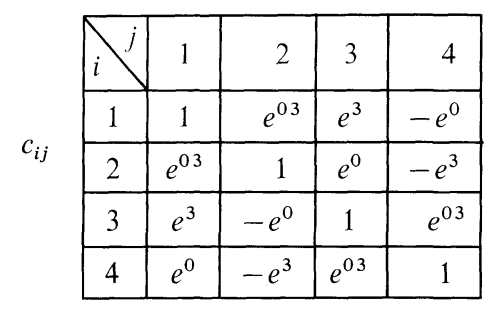

This yields the following matrix basis, $\mathbf{e}_{i j}$.

\begin{tabular}{|c|c|c|c|c|}
\hline$i$ & 1 & 2 & 3 & 4 \\
\hline 1 & $P_{1}$ & $P_{1} e^{03}=e^{03} P_{2}$ & $P_{1} e^{3}=e^{3} P_{3}$ & $-P_{1} e^{0}=-e^{0} P_{4}$ \\
\hline 2 & $e^{03} P_{1}=P_{2} e^{03}$ & $P_{2}$ & $P_{2} e^{0}=e^{0} P_{3}$ & $-P_{2} e^{3}=-e^{3} P_{4}$ \\
\hline 3 & $e^{3} P_{1}=P_{3} e^{3}$ & $-P_{3} e^{0}=-e^{0} P_{2}$ & $P_{3}$ & $P_{3} e^{03}=e^{03} P_{4}$ \\
\hline 4 & $e^{0} P_{1}=P_{4} e^{0}$ & $-P_{4} e^{3}=-e^{3} P_{2}$ & $P_{4} e^{03}=e^{03} P_{3}$ & $P_{4}$ \\
\hline
\end{tabular}

It can be checked that (1.11) is satisfied by

$$
C=e^{123} \text {. }
$$

\section{The Decomposition of the Even Sub-algebra}

The involution $\eta$ is defined by $\eta X=-X$ if $X$ is a covector, and satisfies

$$
\eta S_{p}(\mathscr{A})=(-1)^{p} S_{p}(\mathscr{A})
$$

Elements that are even under $\eta$ form a sub-algebra, $\mathscr{A}^{+}$. If $P^{+}$is a primitive idempotent of $\mathscr{A}^{+}$, then

$$
P^{+} \mathscr{A}^{+} P^{+}=\left\{2 S_{0}\left(\mathscr{A}^{+} P^{+}\right)-2 z S_{0}\left(\mathscr{A}^{+} P^{+} z\right)\right\} P^{+} .
$$

The volume 4-form, $* 1$, is denoted by $z$ and satisfies $z^{2}=-1$. Thus $P^{+} \mathscr{A}^{+} P^{+}$is the algebra of complex numbers, with "imaginary" element $z$ and identity $P^{+}$. (Note that $z$ is in the centre of $\mathscr{A}^{+}$.) The identity in $\mathscr{A}^{+}$can be written as the sum of two orthogonal primitive idempotents, and Wedderburn's theorem then gives

$$
\mathscr{A}^{+}=\mathbb{C} \times \mathscr{M}_{2},
$$

i.e. the algebra of complex $2 \times 2$ matrices. In fact,

$$
\mathscr{A}^{+}=\sum_{\alpha, \beta=1}^{2} A_{\alpha \beta}^{+} \boldsymbol{\varepsilon}_{\alpha \beta},
$$


with

$$
A_{\alpha \beta}^{+}=2 S_{0}\left(\mathscr{A}^{+} \boldsymbol{\varepsilon}_{\beta \alpha}\right)-2 z S_{0}\left(z \mathscr{A}^{+} \boldsymbol{\varepsilon}_{\beta \alpha}\right),
$$

and

$$
\varepsilon_{\alpha \beta}=P_{\alpha}^{+} c_{\alpha \beta}^{+} P_{\beta}^{+},
$$

where

$$
P_{\alpha}^{+}=c_{\alpha \beta}^{+} P_{\beta}^{+} c_{\alpha \beta}^{+-1} .
$$

We denote transposition in $\mathscr{A}^{+}$by ${ }^{t}$, and relate it to $\xi$ by

$$
\mathscr{A}^{+t}=C^{+-1} \xi \mathscr{A}^{+} C^{+} \text {, }
$$

where we have

$$
\xi C^{+}=-C^{+}
$$

for some $C^{+} \in \mathscr{A}^{+}$.

Since primitives in $\mathscr{A}^{+}$are not primitive in $\mathscr{A} \exists x: \eta x=-x$,

$$
x^{2}=1 \quad \text { and } \quad x P_{\alpha}^{+}=P_{\alpha}^{+} x, \quad \forall \alpha .
$$

Then $\left\{P_{\alpha}^{+} \frac{1}{2}(1 \pm x)\right\}$ is a set of pairwise orthogonal primitive idempotents of $\mathscr{A}$. In fact, we may choose $x$ such that

$$
x \varepsilon_{\alpha \beta}=\varepsilon_{\alpha \beta} x, \quad \forall \alpha, \beta .
$$

So if we expand in a matrix basis as in (II.4)

$$
x \mathscr{A}^{+} x=\sum_{\alpha, \beta=1}^{2} A_{\alpha \beta}^{+*} \varepsilon_{\alpha \beta},
$$

since $x z=-z x$. If we follow the approach above to construct the matrix basis for $\mathscr{A}$ then we have

$$
C=x C^{+} .
$$

Thus transposition in $\mathscr{A}$ induces hermitian conjugation in $\mathscr{A}^{+}$. The following complements the previous example.

Let

$$
P_{1}^{+}=P_{1}+P_{3}, \quad P_{2}^{+}=P_{2}+P_{4},
$$

where $P_{1}$ etc. are given by (I.15). Choosing

$$
c_{12}^{+}=e^{03}
$$

gives the following basis, $\boldsymbol{\varepsilon}_{\alpha \beta}$.

\begin{tabular}{|c|c|c|}
\hline$\alpha$ & 1 & 2 \\
\hline 1 & $P_{1}^{+}$ & $P_{1}^{+} e^{03}=e^{03} P_{2}^{+}$ \\
\hline 2 & $e^{03} P_{1}^{+}=P_{2}^{+} e^{03}$ & $P_{2}^{+}$ \\
\hline
\end{tabular}




\section{Inner Products on Ideals}

We can define spin-invariant inner products on the minimal left ideals of $\mathscr{A}$ and $\mathscr{A}^{+}$(cf. [6]). If $\phi=\phi P, \psi=\psi P$, where $P$ is a primitive idempotent in $\mathscr{A}$, then

$$
(\phi, \psi)_{\mathscr{R}} \equiv C^{-1} \xi \phi \psi=4 S_{0}\left(C^{-1} \xi \phi \psi\right) P,
$$

with $C$ as in (I.11) so that $C^{-1} \xi P=P C^{-1}$. (This bracket defines a mapping into the algebra of real numbers with identity $P$. This algebra is trivially mapped into the algebra of real numbers with identity 1 by projecting with $S_{0}$.) As a consequence of (I.14) we have

$$
(\phi, \psi)_{\mathscr{R}}=-(\psi, \phi)_{\mathscr{R}} .
$$

The spin-invariance follows from

$$
(Q \phi, Q \psi)_{\mathscr{R}}=(\phi, \psi)_{\mathscr{R}} \quad \text { if } \quad \xi Q=Q^{-1} .
$$

In fact, elements $Q$ of $\operatorname{PIN}(3,1)$ satisfy $\xi Q=Q^{-1}$ if $V \rightarrow Q V Q^{-1}$ is a space orientation preserving transformation on the space of covectors. (Actually, this corresponds to what is called in the physics literature Racah time-reversal. We intend discussing these discrete transformations in more detail elsewhere.)

Given that $\xi P=C P C^{-1}$ we have

$$
\bar{P}=B P B^{-1},
$$

where $\bar{P} \equiv \xi \eta P, B=z C$. Also

$$
\bar{B}=-B \quad \text { iff } \quad \xi C=-C
$$

(cf. [5]). Proceeding as above we define

and observe

$$
(\phi, \psi)_{\mathscr{P}} \equiv B^{-1} \bar{\phi} \psi=4 S_{0}\left(B^{-1} \bar{\phi} \psi\right) P,
$$

$$
(\phi, \psi)_{\mathscr{P}}=-(\psi, \phi)_{\mathscr{P}}
$$

and

$$
(Q \phi, Q \psi)_{\mathscr{P}}=(\phi, \psi)_{\mathscr{P}} \quad \text { if } \quad \bar{Q}=Q^{-1} .
$$

All time-orientation preserving elements of $\operatorname{PIN}(3,1)$ satisfy $\bar{Q}=Q^{-1}$, and so this is a "parity" invariant inner product. The two inner products are related by

$$
(\phi, \psi)_{\mathscr{P}}=-(\phi, z \psi)_{\mathscr{R}} .
$$

We introduce a spin-invariant inner product on the minimal left ideals of $\mathscr{A}^{+}$ by defining

$$
\langle\alpha, \beta\rangle \equiv C^{+^{-1}} \xi \alpha \beta=\left\{2 S_{0}\left(C^{+-1} \xi \alpha \beta\right)-2 z S_{0}\left(C^{+^{-1}} \xi \alpha \beta z\right)\right\} P^{+}
$$

for $\alpha, \beta \in \mathscr{A}^{+}: \alpha P^{+}=\alpha, \beta P^{+}=\beta, P^{+}$primitive in $\mathscr{A}^{+}$. Spin $(3,1)$ invariance follows since

$$
Q \in \mathscr{A}^{+}: \quad \xi Q=Q^{-1} \quad \text { if } \quad Q \in \operatorname{Spin}(3,1)
$$

(see e.g. [7]). This is the usual symplectic metric on the space of 2-component complex spinors. 


\section{A Majorana "Neutrino" Model}

We now use the preceding formalism to examine a model involving a "Majorana spinor"; that is, a field lying in a minimal left ideal of $\mathscr{A}$. The field equations are obtained from the action-density 4-form

$$
\Lambda=S_{0}\left\{C^{-1} \xi(\phi P) d(\phi P)\right\} z=S_{0}(\phi P, d(\phi P))_{\mathscr{R}} z,
$$

$\phi \in \mathscr{A}$ and $P$ is primitive in $\mathscr{A}$. This is an obvious modification of the Kähler action [3] describing a field lying in one minimal left ideal. [Note that the adjoint properties of $d, S_{0}(\xi \phi d \psi) z=-S_{0}(\xi \psi d \phi) z \bmod d$, together with (I.14) prevent $\Lambda$ being exact without recourse to further anti-commuting parameters.] In the following section we shall show the relation of this action to the Dirac action. The operator $d$ can be written in terms of the pseudo-Riemannian connection,

$$
d=e^{a} \nabla_{X_{a}},
$$

where $\left\{X_{a}\right\}$ is an arbitrary frame and $\left\{e^{a}\right\}$ its dual. Thus (IV.1) contains no preferred frames.

However, it does contain a particular idempotent $P$. All primitive idempotents are similar,

$$
P^{\prime}=S P S^{-1}, \quad C^{\prime}=\xi S^{-1} C S^{-1},
$$

where $S$ is an arbitrary invertible element of $\mathscr{A}$ and $C^{\prime}$ satisfies $C^{\prime-1} \xi P^{\prime} C^{\prime-1}$ $=P^{\prime}$.

We now show that if we supplement these transformations by

$$
\phi^{\prime}=\phi S^{-1},
$$

then $\Lambda^{\prime}=S_{0}\left(C^{\prime-1} \xi\left(\phi^{\prime} P^{\prime}\right) d\left(\phi^{\prime} P^{\prime}\right)\right) z=\Lambda$. For brevity we set

then use of (I.3) readily gives

$$
\psi \equiv \phi P \text {, }
$$

$$
\Lambda^{\prime}-\Lambda=4 S_{0}\left(C^{-1} \xi \psi e^{a} \psi\right) S_{0}\left(P \nabla_{X_{a}} S^{-1} S\right) z .
$$

The first factor vanishes by virtue of (I.13) and (I.14). The covariance given by (IV.3), (IV.4) is a GL $(4, R)$ covariance, since $S$ can be any invertible element. This covariance corresponds to the usual freedom to choose a representation of the $\gamma$-matrices. For we may extend a given $P$ to an orthogonal set $\left\{P_{i}\right\}$, and hence construct a matrix basis $\left\{\mathbf{e}_{i j}\right\}$. If $\left\{\mathbf{e}_{i j}^{\prime}\right\}$ denotes the basis obtained in this way from $P^{\prime}$ then

where

$$
\begin{gathered}
\psi=\sum_{i} \psi^{i} \mathbf{e}_{i 1}, \\
\psi S^{-1}=\sum_{i} S^{-1} \psi^{i} \mathbf{e}_{i 1}^{\prime}=\sum_{i j} S_{i j}^{-1} \psi^{j} \mathbf{e}_{i 1}^{\prime},
\end{gathered}
$$

$$
S^{-1}=\sum_{i j} S_{i j}^{-1} \mathbf{e}_{i j}^{\prime} \text {. }
$$

Thus the transformation which acts from the right in (IV.4) appears as a transformation from the left on the components with respect to the transformed basis.

Up to this point the discussion has placed no restriction on the metric $g$. We now discuss covariance under isometric diffeomorphisms of Minkowski space. If 
$L: M \rightarrow M: L^{*} g=g$, then $\left(L^{*} P\right)^{2}=L^{*} P$ if $P^{2}=P$. Thus

$$
L^{*} P=Q P Q^{-1} \text { for some } Q \text {. }
$$

Since we have already established the invariance of $\Lambda$ under changes of $P$, there is no loss of generality in choosing $P$ such that $Q \in \operatorname{Pin}(3,1)$. Use of this inner automorphism invariance readily gives $L^{*}\{\Lambda(\phi, P, C)\}=\Lambda\left(L^{*} \phi Q, P, C\right)$ if $\xi Q=Q^{-1}$, i.e. if $L$ is space-orientation preserving. If we expand $\psi$ as in (IV.7) and set $Q=\sum_{i j} Q_{i j} \mathbf{e}_{i j}$, then

$$
\begin{aligned}
\psi^{\prime} & \equiv L^{*} \phi Q P \\
& =\sum_{i j} Q_{i j} L^{*} \psi^{j} \mathbf{e}_{i 1} .
\end{aligned}
$$

The Clifford multiplication by $Q$ provides the usual "intrinsic spin" transformation whilst the diffeomorphic image of the components accounts for "orbital" contributions.

We may obtain the field equations for $\phi$ (in a general space time) by taking arbitrary $\phi$ variations of (IV.1). This yields

$$
d(\phi P) P+2 S_{0}\left(P \nabla_{X_{a}} C^{-1} C\right) e^{a} \phi P=0 .
$$

(If we were in Minkowski space with covariantly constant $P, C$ then this would simply correspond to one ideal projected out of the Kähler equation.) We can cast (IV.13) into a more familiar guise.

\section{Suppose}

$$
\exists\left\{\mathbf{e}_{i j}\right\}: \nabla_{X} \mathbf{e}_{i j}=\left[\Sigma_{X}, \mathbf{e}_{i j}\right] V i, j,
$$

where the bracket denotes a Clifford commutator. (The existence of such a basis will be demonstrated shortly.) Then

i.e.

$$
\begin{aligned}
\nabla_{X} \mathbf{e}_{i j}{ }^{\prime} & \equiv \nabla_{X}\left(S \mathbf{e}_{i j} S^{-1}\right) \\
& =\nabla_{X} S S^{-1} \mathbf{e}_{i j}{ }^{\prime}+S\left[\Sigma_{X}, \mathbf{e}_{i j}\right] S^{-1}+\mathbf{e}_{i j}{ }^{\prime} S \nabla_{X} S^{-1} \\
& =\nabla_{X} S S^{-1} \mathbf{e}_{i j}{ }^{\prime}+\left[S \Sigma_{X} S^{-1}, \mathbf{e}_{i j}{ }^{\prime}\right]-\mathbf{e}_{i j}{ }^{\prime} \nabla_{X} S S^{-1},
\end{aligned}
$$

with

$$
\nabla_{X} \mathbf{e}_{i j}{ }^{\prime}=\left[\Sigma_{X}^{\prime}, \mathbf{e}_{i j}{ }^{\prime}\right]
$$

Since $C$ was defined by $\mathbf{e}_{j i}=C^{-1} \xi \mathbf{e}_{i j} C$, we have

$$
\nabla_{X} \mathbf{e}_{j i}=\left[\nabla_{X} C^{-1} C-C^{-1} \xi \Sigma_{X} C, \mathbf{e}_{j i}\right] .
$$

Comparison with (IV.14) gives

$$
\nabla_{X} C^{-1} C=\Sigma_{X}+C^{-1} \xi \Sigma_{X} C,
$$

where the central part of $\Sigma_{X}$ is chosen such that

$$
S_{0}\left(\Sigma_{X}\right)=\frac{1}{2} S_{0}\left(\nabla_{X} C^{-1} C\right) .
$$

Use of (IV.14) and (IV.18) gives the matrix components of (IV.13),

$$
\sum_{a, i} e^{a}\left[X_{a} \psi^{i}+\sum_{X_{a}} \psi^{i}\right] \mathbf{e}_{i 1}=0 .
$$

We have used (IV.5) and (IV.7). 
In this equation $\Sigma_{X_{a}}$ is seen to play the rôle of a connection. We finally make contact with the usual curved space Dirac equation by relating $\Sigma_{X}$ to the pseudo-Riemannian connection. If $\left\{e^{a}\right\}$ is orthonormal then

$$
\nabla_{X} e^{a}=\sum_{p, q} \frac{1}{4} i_{X} \omega_{p q}\left[e^{p q}, e^{a}\right],
$$

where $\left\{\omega_{p q}\right\}$ are the connection 1-forms, and $i_{X}$ is the interior derivative. If

$$
e^{a}=\sum_{i j} \gamma_{i j}^{a} \mathbf{e}_{i j}
$$

with $\gamma_{i j}^{a}$ constants, then $\nabla_{X} e^{a}=\sum_{i j} \gamma_{i j}^{a} \nabla_{X} \mathbf{e}_{i j}$, that is, from (IV.14) and (IV.21) we have chosen a matrix basis such that

$$
\Sigma_{X}=\sum_{p, q} \frac{1}{4} i_{X} \omega_{p q} e^{p q} .
$$

Choosing an orthonormal frame to have constant matrix components correlates the choice of frame with the choice of matrix basis. Thus this condition is only preserved if a change of frame is accompanied by an orthogonal transformation on the matrix basis. This is in accord with the usual rules for the spinorial covariance of the Dirac equation [cf. (IV.8)].

The action (IV.1) is a functional not only of $\phi$ but also of some $P$ (with an associated $C$ ) and the metric. We emphasize that there is no independent connection, the only one present being the pseudo-Riemannian one obtained from the metric structure. In a complete theory it would be desirable that all degrees of freedom be dynamical. If $P$ is taken to be idempotent then variations of $P$ (and $C$ ) for a given metric give no new equations since the established invariance of $\Lambda$ shows that these variations can be compensated by a variation of $\phi$. However, since $P$ is idempotent it is important to correctly treat it in the metric variations that provide the stress tensor which would provide the coupling to gravity. (We will elsewhere demonstrate how this may be accomplished.)

\section{The Dirac Theory}

Throughout we have been concerned with the real Clifford algebra $\mathscr{A}$. As mentioned in the introduction the inclusion of U(1) "charged" spinors is not equivalent to complexifying the Clifford algebra. The Dirac action for a particle of mass $m$ is usually written in terms of a complex spinor. If we choose a Majorana representation in which the $\gamma$-matrices are real then this action can be written in terms of the real and imaginary parts of the spinor. It is straightforward to see that this is equivalent to

$$
\Lambda=S_{0}\left\{B^{-1} \bar{\beta} d \alpha-B^{-1} \bar{\alpha} d \beta-m B^{-1} \bar{\alpha} \beta+m B^{-1} \bar{\beta} \alpha\right\} z,
$$

where $\alpha=\alpha P, \beta=\beta P$ for $P$ primitive in $\mathscr{A}$ and $B$ as in (III.4). $\alpha$ and $\beta$ are usually encoded into the real and imaginary parts of a complex spinor. Of course, that may be done here, but if we follow the analysis of the previous chapter (V.1) will not be invariant under arbitrary inner automorphisms of the complex Clifford algebra. If we set

$$
\lambda=\frac{1}{\sqrt{2}}(\alpha+z \beta), \quad \mu=\frac{1}{\sqrt{2}}(\alpha-z \beta),
$$


then (V.1) can be written

$$
\Lambda=S_{0}\left\{C^{-1} \xi \mu d \mu-C^{-1} \xi \lambda d \lambda+m C^{-1} \xi \lambda \mu-m C^{-1} \xi \mu \lambda\right\} z,
$$

showing the relation of the previous model to the Dirac theory.

Although our algebra is real we have seen in Sect. II how the even sub-algebra is a matrix algebra over the complex field. Since the operator $d$ changes the parity under $\eta$ we can decompose our equation into even and odd components. The field equations obtained from (V.3) are

where

$$
\left.\begin{array}{l}
d \mu P+\omega \mu=m \lambda \\
d \lambda P+\omega \lambda=m \mu
\end{array}\right\}
$$

$$
\omega=2 S_{0}\left(P \nabla_{X_{a}} C^{-1} C\right) e^{a}
$$

If

then

$$
P=\frac{1}{2} P^{+}(1+x)
$$

$$
\mu=u+x u^{*} \quad \text { with } \quad u=u P^{+} \text {and } u^{*} \equiv x u x .
$$

(We use the notation of Sect. II.) This expresses a "Majorana spinor" in terms of two inequivalent "Weyl spinors" that are related by complex conjugation. Similarly,

$$
\lambda=w+x w^{*}, \quad w=w P^{+} .
$$

The odd components of (V.4) are related to the even ones by complex conjugation, so (V.4) can be written as a pair of equations in the even sub-algebra,

$$
\left.\begin{array}{l}
x d u P^{+}+x \Omega u=m w^{*}, \\
d(w x) P^{+}+\Omega(w x)=m u,
\end{array}\right\}
$$

where

$$
\Omega=\omega+e^{a}\left\{S_{0}\left(\nabla_{X_{a}} x x P^{+}\right)-z S_{0}\left(z \nabla_{X_{a}} x x P^{+}\right)\right\} .
$$

In (V.9) we have the Dirac equation written as a pair of coupled equations for two 2-component complex "Weyl" spinors. If we set

$$
\Psi=u+w x,
$$

then the two equations in (V.9) can be added to give

$$
(d \Psi) P^{+}+\Omega \Psi=m \Psi \text {. }
$$

We have $\Psi=\Psi P^{+}$, but $\Psi \notin \mathscr{A}^{+}$, that is, $\Psi$ lies in a non-minimal left ideal of $\mathscr{A}$. It is trivial that if $\Psi$ satisfies (V.12) then so does $\Psi_{z}$. Since $z^{2}=-1$ this is a U(1) covariance; in fact, this is usually identified as the gauge symmetry of electromagnetism. The usual electromagnetic coupling in (V.1) is

$$
\Lambda_{\mathrm{E} . \mathrm{M} .}=S_{0}\left(B^{-1} \bar{\alpha} A \alpha+B^{-1} \bar{\beta} A \beta\right) z,
$$

where $A$ is the Maxwell 1-form. This leads to the interaction modifying (V.12) to

$$
(d \Psi) P^{+}+\Omega \Psi+A \Psi_{z}=m \Psi .
$$


We stress that the encoding of a "charged" spinor into a certain non-minimal ideal and the identification of right multiplication by $z$ with the U(1) of electromagnetism both follow from a faithful correspondence with conventional wisdom. Furthermore (as will be demonstrated elsewhere), this formulation of the electromagnetic coupling leads to a conventional coupling to gravity via the usual stress tensor.

\section{Conclusion}

We have shown in detail the correspondence between an equation for a spinorial section of the Kähler-Atiyah bundle and the usual matrix formulation of the Dirac equation. We believe that this leads to a conceptual simplification: essentially, the spin-frames and spin-connection are explicitly constructed from the exterior bundle and the pseudo-Riemannian connection on space-time. We have shown how the usual spinorial covariance is seen in this light to correspond to correlating the choice of orthonormal frame to choice of ideal. (This corresponds to the view taken in [8].)

The description of the Dirac equation in terms of a real Kähler-Atiyah algebra offers new perspectives for incorporating interactions with other fields. In particular, we expect to investigate in a further paper the stress tensor of an interacting system in this formalism, together with the dynamical status of the idempotents. Certainly, our work above implies that both the Dirac and Kähler field theories are intriguing descriptions of half-integer spin systems in terms of differential forms. Whether or not they are competing descriptions for fundamental fermions can only be ascertained by further scrutiny.

\section{References}

1. Kähler, E.: Der innere Differentialkalkül. Rend. Mat. (3-4) 21, 425 (1962)

2. Graf, W.: Differential forms as spinors. Ann. Inst. Henri Poincaré XXIX, 85 (1978)

3. Benn, I.M., Tucker, R.W.: Fermions without spinors. Commun. Math. Phys. 89, 341 (1983)

4. Albert, A.A.: Structure of algebras. American Mathematical Society Colloquium Publications, Vol. XXIV, 1961

5. Benn, I.M., Tucker, R.W.: Clifford analysis of exterior forms and Fermi-Bose symmetry. J. Phys. A 16, 4147 (1983)

6. Lounesto, P.: Scalar products of spinors and an extension of Brauer-Wall groups. Found. Phys. 11, 721 (1981)

7. Porteus, I.R.: Topological geometry. Cambridge: Cambridge University Press 1981

8. Benn, I.M., Tucker, R.W.: A local right-spin covariant Kähler equation. Phys. Lett. B 130, 177 (1983)

Communicated by S. W. Hawking

Received February 16, 1984; in revised form August 24, 1984 
